\title{
Superspace Unitary Operator in Superfield Approach to Non-Abelian Gauge Theory with Dirac Fields
}

\author{
T. Bhanja, ${ }^{1}$ D. Shukla, ${ }^{1}$ and R. P. Malik ${ }^{1,2}$ \\ ${ }^{1}$ Physics Department, Institute of Science, Banaras Hindu University, Varanasi, Uttar Pradesh 221 005, India \\ ${ }^{2}$ DST Centre for Interdisciplinary Mathematical Sciences, Institute of Science, Banaras Hindu University, Varanasi 221005 , India \\ Correspondence should be addressed to D. Shukla; dheerajkumarshukla@gmail.com
}

Received 28 December 2015; Accepted 26 April 2016

Academic Editor: Edward Sarkisyan-Grinbaum

Copyright (c) 2016 T. Bhanja et al. This is an open access article distributed under the Creative Commons Attribution License, which permits unrestricted use, distribution, and reproduction in any medium, provided the original work is properly cited. The publication of this article was funded by $\mathrm{SCOAP}^{3}$.

\begin{abstract}
Within the framework of augmented version of the superfield approach to Becchi-Rouet-Stora-Tyutin (BRST) formalism, we derive the superspace unitary operator (and its Hermitian conjugate) in the context of four $(3+1)$-dimensional $(4 \mathrm{D})$ interacting nonAbelian 1-form gauge theory with Dirac fields. The ordinary 4D non-Abelian theory, defined on the flat 4D Minkowski spacetime manifold, is generalized onto a $(4,2)$-dimensional supermanifold which is parameterized by the spacetime bosonic coordinates $x^{\mu}$ (with $\mu=0,1,2,3$ ) and a pair of Grassmannian variables $(\theta, \bar{\theta})$ which satisfy the standard relationships: $\theta^{2}=\bar{\theta}^{2}=0$ and $\theta \bar{\theta}+\bar{\theta} \theta=$ 0 . Various consequences of the application of the above superspace (SUSP) unitary operator (and its Hermitian conjugate) are discussed. In particular, we obtain the results of the application of horizontality condition (HC) and gauge-invariant restriction (GIR) in the language of the above SUSP operators. One of the novel results of our present investigation is the derivation of explicit expressions for the SUSP unitary operator (and its Hermitian conjugate) without imposing any Hermitian conjugation condition from outside on the parameters and (super)fields of the supersymmetric version of our 4D interacting non-Abelian 1-form theory with Dirac fields.
\end{abstract}

\section{Introduction}

The covariant canonical quantization of a given gauge theory is performed within the framework of Becchi-Rouet-StoraTyutin (BRST) formalism where the local gauge symmetries of the classical gauge theory are traded with the quantum gauge (i.e., BRST) symmetries. For a single classical local gauge symmetry, there exist two quantum gauge symmetries which are christened as the BRST and anti-BRST symmetries. In one stroke, the BRST formalism provides (i) the covariant canonical quantization, (ii) the proof of unitarity, (iii) the physicality criteria in the quantum Hilbert space, and so forth, for a given gauge theory. The two key mathematical properties, associated with the above (anti-)BRST symmetries, are the nilpotency property and absolute anticommutativity. The former property establishes the fermionic (i.e., supersymmetric) nature of these symmetries and the latter property encodes the linear independence of the BRST and anti-BRST transformations.
The superfield approach (see, e.g., [1-5]) to BRST formalism provides the geometrical origin and interpretation for the nilpotency and absolute anticommutativity properties of the (anti-)BRST symmetries that are required for the covariant canonical quantization of the $p$-form $(p=1,2,3, \ldots)$ gauge theories. In particular, the horizontality condition (HC) plays a pivotal role in the derivation of the nilpotent (anti-)BRST symmetry transformations connected with the $p$-form Abelian gauge and corresponding (anti)ghost fields of the theory and it also leads to the systematic derivation of the Curci-Ferrari (CF) condition(s). The latter have been shown to be connected with the geometrical objects called gerbes in the context of BRST description of the Abelian 2form and Abelian 3-form gauge theories [6, 7] in four $(3+1)$ and six $(5+1)$ dimensions of the flat Minkowskian spacetime, respectively.

The above usual superfield approach [1-5] has been systematically generalized to incorporate the additional restrictions on the superfields so as to derive the proper 
(anti-)BRST symmetries for the matter fields in an interacting gauge theory (in addition to the nilpotent (anti-)BRST symmetries for the gauge and (anti)ghost fields that are derived due to HC). These additional restrictions are found to be consistent with the HC because the geometrical interpretations for the (anti-)BRST symmetries remain intact. In particular, it is the gauge-invariant restrictions involving the covariant derivatives on the matter fields that play a decisive role in this direction where the inputs from the $\mathrm{HC}$ also play a key role. We have christened these generalized versions of superfield approach to BRST formalism as the augmented version of superfield formalism [8-10]. The latter (i.e., the augmented version of superfield formalism) is basically the consistent generalization of the usual superfield formalism (particularly developed in [1-3]).

A superspace (SUSP) unitary operator has been shown to exist in the superfield approach to the description of 4D nonAbelian 1-form gauge theory within the framework of BRST formalism (see, e.g., [1-3]). The beauty of this operator is the observation that it maintains the explicit $\mathrm{SU}(N)$ group structure in the transformation space of the superspace (where the ordinary fields, defined on the $D$-dimensional ordinary flat Minkowskian manifold, are generalized onto the superfields defined on the the $(D, 2)$-dimensional supermanifold). This explicit group structure is somewhat hidden in the direct application of HC. Thus, the utility of the SUSY unitary operator has an edge over the utility of $\mathrm{HC}$ because the results of the latter could be derived by using the former one (cf. Section 5). The central theme of our present paper is to derive this unitary operator (and its Hermitian conjugate) explicitly by applying the augmented version of superfield formalism [8-10] in the description of the $4 \mathrm{D}$ non-Abelian interacting theory with Dirac fields. It should be noted that this operator has been judiciously chosen in [1-3] for the BRST description of the $4 \mathrm{D}$ interacting non-Abelian gauge theory with a generic matter field. One of the central aims of our present investigation is to theoretically prove the sanctity and preciseness of this choice.

Our present investigation is motivated by the following key factors. First, the explicit computation of the SUSP unitary operator $U(x, \theta, \bar{\theta})$ in $\psi(x) \rightarrow \Psi(x, \theta, \bar{\theta})=U(x, \theta$, $\bar{\theta}) \psi(x)$ maintains the $\mathrm{SU}(N)$ group structure in the transformation space. Second, the existence of this $S U(N)$ group structure leads to the definition of the supercovariant derivative on the superfield which obeys the same transformation property (i.e., $D \psi \rightarrow \widetilde{D} \Psi=U(x, \theta, \bar{\theta}) D \psi)$. Third, the definition of the covariant derivative and its transformation rule yield the transformation rule for the supercurvature 2-form (i.e., $\left.D D \psi \rightarrow \widetilde{D} \widetilde{D} \Psi=U(D D \psi) \Rightarrow \widetilde{F}^{(2)}=U F^{(2)} U^{\dagger}\right)$. Fourth, the above arguments lead to the results that are obtained only due to the application of HC (cf. Section 3 below). Thus, in some sense, the derivation of the SUSP operators $U(x, \theta, \bar{\theta})$ and $U^{\dagger}(x, \theta, \bar{\theta})$ provides an alternative to the $\mathrm{HC}$ where $\mathrm{SU}(N)$ group structure is respected very clearly. Fifth, we theoretically derive the SUSP unitary operator and mathematically support the sanctity of the choice made in [1$3]$. The judicious choice made in reference [1-3] is somewhat straightforward when one knows the (anti-)BRST symmetry transformations beforehand. However, the explicit derivation, within the framework of our superfield formalism, is a novel result for the 4D non-Abelian 1-form gauge theory with Dirac fields. Finally, our present attempt is the generalization of our earlier work on the 4D Abelian 1-form interacting gauge theory with Dirac and complex scalar fields [11] to the $4 \mathrm{D}$ interacting non-Abelian 1-form gauge theory with Dirac fields.

The contents of our present investigation are organized as follows. To set up the notations and convention, in Section 2, we briefly recapitulate the bare essentials of the (anti-)BRST symmetries for the 4D interacting non-Abelian gauge theory with Dirac fields in the Lagrangian formulation. Section 3 is devoted to a concise discussion on the horizontality condition (HC) and the derivation of the nilpotent (anti-)BRST symmetries for the gauge and (anti)ghost fields. In Section 4, we derive the nilpotent (anti-)BRST symmetry transformations for the Dirac fields by exploiting the power and potential of gauge-invariant restriction (GIR) on the superfields defined on the $(4,2)$-dimensional supermanifold. Section 5 contains the derivation of SUSP unitary operator and it incorporates various consequences that emerge out from the knowledge of this unitary operator (and its Hermitian conjugate). Finally, in Section 6, we summarize our key results and point out a few future directions for further investigations. In Appendix, we discuss a few things for readers' convenience.

Convention and Notations. We adopt the convention and notations such that the 4D Minkowskian flat spacetime manifold is endowed with a metric $\eta_{\mu \nu}$ with signatures $(+1,-1,-1,-1)$ so that $\partial_{\mu} A^{\mu}=\eta_{\mu \nu} \partial^{\mu} A^{\nu}=\partial_{0} A_{0}-\partial_{i} A_{i}$, where the Greek indices $\mu, \nu, \lambda, \ldots=0,1,2,3$ correspond to the spacetime directions and Latin indices $i, j, k, \ldots=$ $1,2,3$ stand for the space directions only. In the Lie-algebraic space, we take dot and cross products between $P^{a}$ and $Q^{a}$ as follows: $P \cdot Q=P^{a} Q^{a}$ and $(P \times Q)^{a}=f^{a b c} P^{b} Q^{c}$, where $f^{a b c}$ are the structure constants that can be chosen to be totally antisymmetric for the $\mathrm{SU}(N)$ group with the Lie algebra $\left[T^{a}, T^{b}\right]=f^{a b c} T^{c}$. Here, $T^{a}$ (with $a=1,2,3, \ldots, N^{2}-$ 1) are the generators of the Lie algebra corresponding to the $\mathrm{SU}(N)$ group. We have used the same notation for the covariant derivative and the dimensionality of the spacetime. However, the different meanings, attached with the symbol $D$, are quite unambiguous in the whole body of the text of our present endeavor. We have explicitly taken the covariant derivatives on the matter field and ghost fields as follows: $D_{\mu} \psi=\left(\partial_{\mu}+i A_{\mu} \cdot T\right) \psi$ and $D_{\mu} C=\partial_{\mu} C+i\left(A_{\mu} \times C\right)$ which are in the fundamental and adjoint representations, respectively.

\section{Preliminaries: (Anti-)BRST Symmetries}

We begin with the (anti-)BRST invariant Lagrangian densities $\mathscr{L}_{B}$ and $\mathscr{L}_{\bar{B}}$ (see, e.g., $\left.[12,13]\right)$ for the $4 \mathrm{D}$ interacting nonAbelian 1-form gauge theory with the massive (with mass $m$ ) Dirac fields $(\psi, \bar{\psi})$, in the Curci-Ferrari gauge (where $\xi=2$ ) $[14,15]$, as 


$$
\begin{aligned}
\mathscr{L}_{B}= & -\frac{1}{4} F_{\mu \nu} \cdot F^{\mu \nu}+\bar{\psi}\left(i \gamma^{\mu} D_{\mu}-m\right) \psi \\
& +s_{b} s_{a b}\left(\frac{i}{2} A_{\mu} \cdot A^{\mu}-\frac{\xi}{2} \bar{C} \cdot C\right) \\
\equiv & -\frac{1}{4} F_{\mu \nu} \cdot F^{\mu \nu}+\bar{\psi}\left(i \gamma^{\mu} D_{\mu}-m\right) \psi+B \cdot\left(\partial_{\mu} A^{\mu}\right) \\
& +\frac{1}{2}(B \cdot B+\bar{B} \cdot \bar{B})-i \partial_{\mu} \bar{C} \cdot D^{\mu} C, \\
\mathscr{L}_{B}= & -\frac{1}{4} F_{\mu \nu} \cdot F^{\mu \nu}+\bar{\psi}\left(i \gamma^{\mu} D_{\mu}-m\right) \psi \\
& -s_{a b} s_{b}\left(\frac{i}{2} A A_{\mu} \cdot A^{\mu}-\frac{\xi}{2} \bar{C} \cdot C\right) \\
\equiv & -\frac{1}{4} F_{\mu \nu} \cdot F^{\mu \nu}+\bar{\psi}\left(i \gamma^{\mu} D_{\mu}-m\right) \psi-\bar{B} \cdot\left(\partial_{\mu} A^{\mu}\right) \\
& +\frac{1}{2}(B \cdot B+\bar{B} \cdot \bar{B})-i D_{\mu} \bar{C} \cdot \partial^{\mu} C,
\end{aligned}
$$

for the explicit derivation of the gauge-fixing and FaddeevPopov ghost terms. In other words, we have derived the final expressions for the Lagrangian densities $\mathscr{L}_{B}$ and $\mathscr{L}_{\bar{B}}$ by taking into account the Curci-Ferrari gauge where $\xi=2$. It will be noted that the above Lagrangian densities $\mathscr{L}_{B}$ and $\mathscr{L}_{\bar{B}}$ are equivalent on the constrained hypersurface defined by the field equation: $B+\bar{B}=-(C \times \bar{C})$ which is nothing but the Curci-Ferrari (CF) condition [16]. In the above, we have the curvature tensor $F_{\mu \nu}=\partial_{\mu} A_{\nu}-\partial_{\nu} A_{\mu}+i\left(A_{\mu} \times A_{\nu}\right)$ which is derived from the 2 -from $F^{(2)}=d A^{(1)}+i A^{(1)} \wedge A^{(1)}$ where the connection 1 -from $A^{(1)}=d x^{\mu}\left(A_{\mu} \cdot T\right)$ defines the vector potential $A_{\mu}^{a}$ in the $\mathrm{SU}(N)$ Lie-algebraic space (with $\left.a, b, c, \ldots=1,2,3, \ldots, N^{2}-1\right)$. The fields $B(x)$ and $\bar{B}(x)$ are the Nakanishi-Lautrup auxiliary fields and the fermionic $\left[\left(C^{a}\right)^{2}=\left(\bar{C}^{a}\right)^{2}=0, C^{a} \bar{C}^{b}+\bar{C}^{b} C^{a}=0\right.$, etc. $]$ (anti)ghost fields $\left(\bar{C}^{a}\right) C^{a}$ are required for the validity of unitarity in the theory. We have a covariant derivative on the Dirac field $\psi$ as follows: $D_{\mu} \psi=\left(\partial_{\mu}+i g A_{\mu} \cdot T\right) \psi \equiv\left(\partial_{\mu}+i A_{\mu} \cdot T\right) \psi$, where the coupling constant $g$ has been set equal to one for the sake of brevity. Similarly, we have taken into consideration the definition of the covariant derivatives (with $g=1$ ) on the (anti)ghost fields as follows: $D_{\mu} \bar{C}=\partial_{\mu} \bar{C}+i\left(A_{\mu} \times \bar{C}\right)$ and $D_{\mu} C=\partial_{\mu} C+i\left(A_{\mu} \times C\right)$. The Dirac fields are fermionic $\left(\psi^{2}=\bar{\psi}^{2}=0, \psi \bar{\psi}+\bar{\psi} \psi=0\right)$ in nature because they commute $\left(\psi A_{\mu}-A_{\mu} \psi=0, \psi B-B \psi=0\right.$, etc. $)$ with all the bosonic fields (e.g., $A_{\mu}, B, \bar{B}$, and $\left.F_{\mu \nu}\right)$ but they anticommute $\left(\psi C^{a}+C^{a} \psi=\right.$ $0, C^{a} \bar{\psi}+\bar{\psi} C^{a}=0, \bar{C}^{a} \psi+\psi \bar{C}^{a}=0$, etc.) with the fermionic (anti)ghost fields $\left(\bar{C}^{a}\right) C^{a}$ of our theory. The above Lagrangian densities (1) respect the following off-shell nilpotent $\left(s_{(a) b}^{2}=\right.$ $0)$, absolutely anticommuting $\left(s_{b} s_{a b}+s_{a b} s_{b}=0\right)$, continuous, and infinitesimal (anti-)BRST symmetry transformations $s_{(a) b}$ (see, e.g., [17] and our Appendix for details):

$$
\begin{aligned}
s_{a b} A_{\mu} & =D_{\mu} \bar{C}, \\
s_{a b} \bar{C} & =-\frac{i}{2}(\bar{C} \times \bar{C}),
\end{aligned}
$$

$$
\begin{aligned}
s_{a b} C & =i \bar{B}, \\
s_{a b} F_{\mu \nu} & =i\left(F_{\mu \nu} \times \bar{C}\right), \\
s_{a b} B & =i(B \times \bar{C}), \\
s_{a b} \bar{B} & =0, \\
s_{a b} \psi & =-i(\bar{C} \cdot T) \psi, \\
s_{a b} \bar{\psi} & =-i \bar{\psi}(\bar{C} \cdot T), \\
s_{b} A \mu & =D \mu, \\
s_{b} C & =-\frac{i}{2}(C \times C), \\
s_{b} \bar{C} & =i B, \\
s_{b} F_{\mu \nu} & =i\left(F_{\mu \nu} \times C\right), \\
s_{b} B & =0, \\
s_{b} \bar{B} & =i(\bar{B} \times C), \\
s_{b} \psi & =-i(C \cdot T) \psi, \\
s_{b} \bar{\psi} & =-i \bar{\psi}(C \cdot T) .
\end{aligned}
$$

The noteworthy points, at this stage, are as follows: (i) The kinetic term $\left[(-1 / 4) F^{\mu \nu} \cdot F_{\mu \nu}\right]$ remains invariant under the (anti-)BRST symmetry transformations. Geometrically, the curvature tensor $F_{\mu \nu}$ has its origin in the exterior derivative $d$ because $F^{(2)}=d A^{(1)}+i A^{(1)} \wedge A^{(1)}$ defines the $F_{\mu \nu}$ tensor (which can be explicitly written in terms of the potential $A_{\mu}$ as follows: $F_{\mu \nu}=\partial_{\mu} A_{\nu}-\partial_{\nu} A_{\mu}+i\left[A_{\mu}, A_{\nu}\right]$, where we observe that $F_{\mu \nu}=F_{\mu \nu} \cdot T$ and $\left.A_{\mu}=A_{\mu} \cdot T\right)$. (ii) The CF-condition remains invariant (i.e., $s_{(a) b}[B+\bar{B}+(C \times \bar{C})]=0$ ) under the offshell nilpotent (anti-)BRST symmetry transformations. Thus, it is a physical constraint that can be imposed on the $4 \mathrm{D}$ nonAbelian interacting 1-form gauge theory. (iii) The Lagrangian densities $\mathscr{L}_{B}$ and $\mathscr{L}_{\bar{B}}$ both are equivalent on the hypersurface in the $4 \mathrm{D}$ Minkowskian spacetime manifold due to the CFcondition (i.e., $B+\bar{B}+(C \times \bar{C})=0)$. In fact, it is on this hypersurface that the off-shell nilpotency and absolute anticommutativity properties of the (anti-)BRST symmetry transformations are valid (see, e.g., [12-15]) in an explicit manner.

\section{Symmetries for the Gauge and (Anti)Ghost Fields: Horizontality Condition}

In this section, we shall exploit the geometrical beauty of $F^{(2)}=d A^{(1)}+i A^{(1)} \wedge A^{(1)}$ in the context of $\mathrm{HC}$ within the framework of superfield formalism and derive the CFcondition: $B+\bar{B}+(C \times \bar{C})=0$ as well as the proper (anti-)BRST symmetry transformations for the gauge and (anti)ghost fields of the $4 \mathrm{D}$ non-Abelian 1-form gauge theory. For this 
purpose, first of all, we define the supercurvature 2-form $\widetilde{F}^{(2)}$ on the $(4,2)$-dimensional supermanifold $\widetilde{F}^{(2)}=\widetilde{d} \widetilde{A}^{(1)}+i \widetilde{A}^{(1)} \wedge$ $\widetilde{A}^{(1)}=(1 / 2)\left(d Z^{M} \wedge d Z^{N}\right) \widetilde{F}_{M N}(x, \theta, \bar{\theta})$, where $Z^{M}=\left(x^{\mu}, \theta, \bar{\theta}\right)$ is the superspace coordinate that characterizes the $(4,2)$ dimensional supermanifold with $x^{\mu}(\mu=0,1,2,3)$ as the bosonic variables and $(\theta, \bar{\theta})$ as a pair of fermionic $\left(\theta^{2}=\bar{\theta}^{2}=0\right.$ and $\theta \bar{\theta}+\bar{\theta} \theta=0)$ Grassmannian variables. The other symbols of relevance, on the appropriately chosen $(4,2)$-dimensional supermanifold, are as follows [1-3, 17]:

$$
\begin{aligned}
d & =d x^{\mu} \partial_{\mu} \longrightarrow \widetilde{d}=d Z^{M} \partial_{M} \\
& =d x^{\mu} \partial_{\mu}+d \theta \partial_{\theta}+d \bar{\theta} \partial_{\bar{\theta}}, \\
A^{(1)} & =d x^{\mu} A_{\mu} \longrightarrow \widetilde{A}^{(1)}=d Z^{M} A_{M} \\
& =d x^{\mu} B_{\mu}+d \theta \bar{F}+d \bar{\theta} F,
\end{aligned}
$$

where $A_{M}=\left(B_{\mu}(x, \theta, \bar{\theta}), F(x, \theta, \bar{\theta}), \bar{F}(x, \theta, \bar{\theta})\right)$ constitutes the vector multiplet and the superspace derivative $\partial_{M}$ stands for $\left(\partial_{\mu}, \partial_{\theta}, \partial_{\bar{\theta}}\right)$. The latter (i.e., $\left.\partial_{M}\right)$ are the components of the superspace derivative $\left(\partial_{M}=\partial / \partial Z^{M}\right)$ with $Z^{M}=\left(x^{\mu}, \theta, \bar{\theta}\right)$.

The requirement of the $\mathrm{HC}$ is to set all the Grassmannian components of $\widetilde{F}_{M N}(x, \theta, \bar{\theta})=\left(\widetilde{F}_{\mu \theta}, \widetilde{F}_{\mu \bar{\theta}}, \widetilde{F}_{\theta \bar{\theta}}, \widetilde{F}_{\theta \theta}, \widetilde{F}_{\bar{\theta} \bar{\theta}}\right)$ equal to zero (i.e., $\widetilde{F}_{\mu \theta}=\widetilde{F}_{\mu \bar{\theta}}=\widetilde{F}_{\theta \bar{\theta}}=\widetilde{F}_{\theta \theta}=\widetilde{F}_{\bar{\theta} \bar{\theta}}=$ $0)$. We know that the kinetic term $\left[-(1 / 4) F^{\mu \nu} \cdot F_{\mu \nu}\right]$ for the gauge field is (anti-)BRST invariant quantity. The $\mathrm{HC}$ basically demands the independence of this quantity (i.e., $\left.-(1 / 4) \widetilde{F}_{M N} \cdot \widetilde{F}^{M N}=-(1 / 4) F_{\mu \nu} \cdot F^{\mu \nu}\right)$. In other words, this gauge- (i.e., BRST-) invariant quantity should be independent of the Grassmannian variables because the latter cannot be realized physically in the ordinary space. This condition leads to the derivation of the relationships between the basic and the auxiliary fields of the Lagrangian densities $\mathscr{L}_{B}$ and $\mathscr{L}_{\bar{B}}$ and the secondary fields $\left(R_{\mu}, \bar{R}_{\mu}, S_{\mu}, \bar{B}_{1}, B_{1}, \bar{B}_{2}, B_{2}, s, \bar{s}\right)$ of the following expansions (see, e.g., [1-3] for details):

$$
\begin{aligned}
B_{\mu}(x, \theta, \bar{\theta}) & =A_{\mu}(x)+\theta \bar{R}_{\mu}(x)+\bar{\theta} R_{\mu}(x)+i \theta \bar{\theta} S_{\mu}(x), \\
F(x, \theta, \bar{\theta}) & =C(x)+i \theta \bar{B}_{1}(x)+i \bar{\theta} B_{1}(x)+i \theta \bar{\theta} s(x), \\
\bar{F}(x, \theta, \bar{\theta}) & =\bar{C}(x)+i \theta \bar{B}_{2}(x)+i \bar{\theta} B_{2}(x)+i \theta \bar{\theta} \bar{s}(x),
\end{aligned}
$$

where, as pointed out earlier, the superfields $B_{\mu}(x, \theta, \bar{\theta})$, $F(x, \theta, \bar{\theta})$, and $\bar{F}(x, \theta, \bar{\theta})$ constitute the multiplet of the vector superfield $A_{M}(x, \theta, \bar{\theta})$. The above expansions are nothing but the shift transformations for the superfields along the Grassmannian directions $(1, \theta, \bar{\theta}, \theta \bar{\theta})$ of the $(4,2)$-dimensional supermanifold. The HC (i.e., $\widetilde{F}_{\theta \bar{\theta}}=\widetilde{F}_{\theta \theta}=\widetilde{F}_{\bar{\theta} \bar{\theta}}=\widetilde{F}_{\mu \theta}=$ $\widetilde{F}_{\mu \bar{\theta}}=0$ ) yields the following interesting and very useful relationships (with $\bar{B}_{1}=\bar{B}, B_{2}=B$ ) $[1-3,17]$ :

$$
\begin{aligned}
& R_{\mu}=D_{\mu} C, \\
& \bar{R}_{\mu}=D_{\mu} \bar{C},
\end{aligned}
$$

$$
\begin{aligned}
B_{1} & =-\frac{1}{2}(C \times C), \\
S_{\mu} & =D_{\mu} B_{2}+D_{\mu} C \times \bar{C} \equiv-D_{\mu} \bar{B}_{1}-D_{\mu} \bar{C} \times C, \\
\bar{B}_{2} & =-\frac{1}{2}(\bar{C} \times \bar{C}), \\
s & =i\left(\bar{B}_{1} \times C\right), \\
\bar{s} & =-i\left(B_{2} \times \bar{C}\right), \\
\bar{B}_{1}+B_{2} & =-(C \times \bar{C}) \longrightarrow B+\bar{B}=-(C \times \bar{C}),
\end{aligned}
$$

where $D_{\mu} C=\partial_{\mu} C+i\left(A_{\mu} \times C\right)$ and the last entry, in the above, is nothing but the CF-condition. The substitution of the above expressions for the secondary fields into (4) yields the following:

$$
\begin{aligned}
B_{\mu}^{(h)}(x, \theta, \bar{\theta})= & A_{\mu}+\theta\left(D_{\mu} \bar{C}\right)+\bar{\theta}\left(D_{\mu} C\right) \\
& +\theta \bar{\theta}\left[i\left(D_{\mu} B+\left(D_{\mu} C \times \bar{C}\right)\right)\right] \\
\equiv & A_{\mu}+\theta\left(s_{a b} A_{\mu}\right)+\bar{\theta}\left(s_{b} A_{\mu}\right) \\
& +i \theta \bar{\theta}\left(s_{b} s_{a b} A_{\mu}\right), \\
F^{(h)}(x, \theta, \bar{\theta})= & C(x)+\theta(i \bar{B})+\bar{\theta}\left(-\frac{i}{2} C \times C\right) \\
& +\theta \bar{\theta}(-\bar{B} \times C) \\
\equiv & C+\theta\left(s_{a b} C\right)+\bar{\theta}\left(s_{b} C\right)+\theta \bar{\theta}\left(s_{b} s_{a b} C\right), \\
\bar{F}^{(h)}(x, \theta, \bar{\theta})= & \bar{C}(x)+\theta\left(-\frac{i}{2} \bar{C} \times \bar{C}\right)+\bar{\theta}(i B) \\
& +\theta \bar{\theta}(B \times \bar{C}) \\
\equiv & \bar{C}(x)+\theta\left(s_{a b} \bar{C}\right)+\bar{\theta}\left(s_{b} \bar{C}\right) \\
& +\theta \bar{\theta}\left(s_{b} s_{a b} \bar{C}\right),
\end{aligned}
$$

where the superscript $(h)$ denotes the expansions obtained after the application of HC. It is to be noted that the CFcondition $B+\bar{B}+(C \times \bar{C})=0$ emerges out from setting the coefficient of $(d \theta \wedge d \bar{\theta})$ equal to zero in the relationship $\widetilde{d} \widetilde{A}^{(1)}+$ $i \widetilde{A}^{(1)} \wedge \widetilde{A}^{(1)}$ which results in the condition $\partial_{\theta} F^{(h)}+\partial_{\bar{\theta}} \bar{F}^{(h)}-$ $i\left\{F^{(h)}, \bar{F}^{(h)}\right\}=0$. It is elementary to check that the explicit substitutions from (6) into this restriction yield the CFcondition $(B+\bar{B}+(C \times \bar{C})=0)$. The (anti-)BRST invariance of the CF-condition can be captured within the framework of superfield formalism as it can be readily checked that $\left.\partial_{\theta}\left[\partial_{\theta} F^{(h)}+\partial_{\bar{\theta}} \bar{F}^{(h)}-i\left\{F^{(h)}, \bar{F}^{(h)}\right\}\right]\right|_{\bar{\theta}=0}=0$ and $\partial_{\bar{\theta}}\left[\partial_{\theta} F^{(h)}+\right.$ $\left.\partial_{\bar{\theta}} \bar{F}^{(h)}-i\left\{F^{(h)}, \bar{F}^{(h)}\right\}\right]\left.\right|_{\theta=0}=0$ which physically imply the (anti-)BRST invariance $\left(s_{(a) b}[B+\bar{B}+(C \times \bar{C})]=0\right)$ of the CF-condition defined on the ordinary $4 \mathrm{D}$ Minkowskian spacetime manifold as the constrained field equation. 


\section{4. (Anti-)BRST Symmetries for the Matter Fields: Gauge-Invariant Restriction}

We exploit the strength of the gauge-invariant restriction (GIR) on the superfields, defined on the $(4,2)$-dimensional supermanifold, to obtain the proper (anti-)BRST symmetry transformations for the matter fields of the interacting $4 \mathrm{D}$ non-Abelian gauge theory with Dirac fields. This appropriate condition (which incorporates the results of $\mathrm{HC}(\mathrm{cf} .(6))$ ) is as follows [17]:

$$
\begin{gathered}
\bar{\Psi}(x, \theta, \bar{\theta})\left(\tilde{d}+i \widetilde{A}_{(h)}^{(1)}\right) \Psi(x, \theta, \bar{\theta}) \\
=\bar{\psi}(x)\left(d+i A^{(1)}\right) \psi(x),
\end{gathered}
$$

where the super 1-form connection on the 1.h.s., derived after the application of $\mathrm{HC}$, is $\widetilde{A}_{(h)}^{(1)}=d x^{\mu} B_{\mu}^{(h)}(x, \theta, \bar{\theta})+$ $d \theta \bar{F}^{(h)}(x, \theta, \bar{\theta})+d \bar{\theta} F^{(h)}(x, \theta, \bar{\theta})$ and the superfields $\Psi(x, \theta, \bar{\theta})$ and $\bar{\Psi}(x, \theta, \bar{\theta})$ have the following expansions along the Grassmannian directions $(\theta, \bar{\theta})$ of the appropriately chosen $(4,2)$ dimensional supermanifold (see, e.g., [17]):

$$
\begin{aligned}
& \Psi(x, \theta, \bar{\theta})=\psi(x)+i \theta \bar{b}_{1}(x)+i \bar{\theta} b_{1}(x)+i \theta \bar{\theta} f(x), \\
& \Psi(x, \theta, \bar{\theta})=\bar{\psi}(x)+i \theta \bar{b}_{2}(x)+i \bar{\theta} b_{2}(x)+i \theta \bar{\theta} \bar{f}(x) .
\end{aligned}
$$

Here, $b_{1} \equiv b_{1} \cdot T, b_{2} \equiv b_{2} \cdot T, \bar{b}_{1} \equiv \bar{b}_{1} \cdot T$, and $\bar{b}_{2} \equiv \bar{b}_{2} \cdot T$ are the bosonic secondary fields in the above expansions and $f \equiv f \cdot T$ and $\bar{f} \equiv \bar{f} \cdot T$ are the fermionic secondary fields. It is obvious that the above superfields are the generalizations of the ordinary 4D fermionic Dirac fields $\psi(x)$ and $\bar{\psi}(x)$ onto the $(4,2)$-dimensional supermanifold because, in the limit $\theta=\bar{\theta}=0$, we retrieve the latter fields from the above superfield expansions. We also note that the r.h.s. of the GIR (7) is a gauge-invariant quantity. Furthermore, the decisive feature of GIR (7) is the key observation that this relationship blends the ideas of $\mathrm{HC}$ and GIR together in a meaningful manner where the expansions of $\left(B_{\mu}^{(h)}(x, \theta, \bar{\theta}), F^{(h)}(x, \theta, \bar{\theta})\right.$, and $\left.\bar{F}^{(h)}(x, \theta, \bar{\theta})\right)($ cf. (6)) play very important role (as the super 1-form $\widetilde{A}_{(h)}^{(1)}=d x^{\mu} B_{\mu}^{(h)}(x, \theta, \bar{\theta})+d \theta \bar{F}^{(h)}(x, \theta, \bar{\theta})+$ $d \bar{\theta} F^{(h)}(x, \theta, \bar{\theta})$ contains these results from the $\mathrm{HC}$ and it appears on the 1.h.s. of the GIR (7)). Thus, we note that (7) incorporates the results of $\mathrm{HC}$.

Taking into account the expansions from (8) and the expressions obtained after the application of the HC (cf. (6)), we obtain the following relationships [17] between the secondary fields of (8) and the basic and auxiliary fields of the Lagrangian densities $\mathscr{L}_{B}$ and $\mathscr{L}_{\bar{B}}$ :

$$
\begin{aligned}
& b_{1}=-(C \cdot T) \psi, \\
& \bar{b}_{1}=-(\bar{C} \cdot T) \psi,
\end{aligned}
$$

$$
\begin{aligned}
& b_{2}=-\bar{\psi}(C \cdot T), \\
& \bar{b}_{2}=-\bar{\psi}(\bar{C} \cdot T), \\
& f=-i[B+\bar{C} C] \psi, \\
& \bar{f}=i \bar{\psi}[B+C \bar{C}] .
\end{aligned}
$$

It will be noted that this is slightly different from the results obtained in [15] for the values of $f$ and $\bar{f}$. However, our present results and relationships in (9) are correct. The substitution of the above values of the secondary fields into expansions (8) yields the following:

$$
\begin{aligned}
\Psi^{(g)}(x, \theta, \bar{\theta})= & \psi(x)+\theta(-i \bar{C} \cdot T) \psi+\bar{\theta}(-i C \cdot T) \psi \\
& +\theta \bar{\theta}(B+\bar{C} C) \psi \\
= & \psi(x)+\theta\left(s_{a b} \psi\right)+\bar{\theta}\left(s_{b} \psi\right) \\
& +\theta \bar{\theta}\left(s_{b} s_{a b} \psi\right), \\
\bar{\Psi}^{(g)}(x, \theta, \bar{\theta})= & \bar{\psi}(x)+\theta[\bar{\psi}(-i \bar{C} \cdot T)] \\
& +\bar{\theta}[\bar{\psi}(-i C \cdot T)] \\
& +\theta \bar{\theta}[\bar{\psi}(-B-C \bar{C})] \\
= & \bar{\psi}(x)+\theta\left(s_{a b} \bar{\psi}\right)+\bar{\theta}\left(s_{b} \bar{\psi}\right) \\
& +\theta \bar{\theta}\left(s_{b} s_{a b} \bar{\psi}\right),
\end{aligned}
$$

where the superscript $(g)$ denotes the superfield expansions after the application of GIR. Thus, we observe that the coefficients of $\theta, \bar{\theta}$ and $\theta \bar{\theta}$ yield the symmetry transformations corresponding to $s_{a b}, s_{b}$ and $s_{b} s_{a b}$, respectively. In other words, we have obtained a relationship between the translational generators $\left(\partial_{\theta}, \partial_{\bar{\theta}}\right)$ along the Grassmannian directions $(\theta, \bar{\theta})$ of the (4,2)-dimensional supermanifold and the (anti-)BRST transformations $s_{(a) b}$ for the matter fields $\psi$ and $\bar{\psi}$ in the ordinary 4D spacetime (cf. (2)). The appropriate mapping between these quantities of interest is as follows: $s_{b} \psi=$ $\left.\partial_{\bar{\theta}} \Psi^{(g)}(x, \theta, \bar{\theta})\right|_{\theta=0}, s_{a b} \psi=\left.\partial_{\theta} \Psi^{(g)}(x, \theta, \bar{\theta})\right|_{\bar{\theta}=0}$, and $s_{b} s_{a b} \psi=$ $\partial_{\bar{\theta}} \partial_{\theta} \Psi^{(g)}(x, \theta, \bar{\theta})$.

\section{SUSP Unitary Operator: Key Consequences}

It is clear from expansions (10) that we have already derived the (anti-)BRST symmetry transformations $s_{(a) b}$ for the Dirac fields which turn out to be the coefficients of $(\theta) \bar{\theta}$ in these expansions. Furthermore, the coefficients of $(\theta \bar{\theta})$ turn out to be $\left(s_{b} s_{a b} \psi\right)$ and $\left(s_{b} s_{a b} \bar{\psi}\right)$, respectively. These expansions (cf. (10)) can be reexpressed as follows:

$$
\begin{aligned}
\Psi^{(g)} & (x, \theta, \bar{\theta}) \\
\quad= & {[1+\theta(-i \bar{C})+\bar{\theta}(-i C)+\theta \bar{\theta}(B+\bar{C} C)] \psi(x) }
\end{aligned}
$$




$$
\begin{aligned}
& \equiv U(x, \theta, \bar{\theta}) \psi(x), \\
\bar{\Psi}^{(g)} & (x, \theta, \bar{\theta}) \\
& =\bar{\psi}(x)[1+\theta(i \bar{C})+\bar{\theta}(i C)+\theta \bar{\theta}(-B-C \bar{C})] \\
& \equiv \bar{\psi}(x) U^{\dagger}(x, \theta, \bar{\theta}),
\end{aligned}
$$

where all the fields are defined in the Lie-algebraic space (e.g., $C=C \cdot T, \bar{C}=\bar{C} \cdot T, B=B \cdot T$, and $\bar{B}=\bar{B} \cdot T)$. Thus, it is obvious that we have derived the SUSP unitary operator $U(x, \theta, \bar{\theta})$ and its Hermitian conjugate $U^{\dagger}(x, \theta, \bar{\theta})$ in a very natural fashion. It is elementary to check that the criterion for the unitarity condition is satisfied by the above SUSP operators:

$$
U(x, \theta, \bar{\theta}) U^{\dagger}(x, \theta, \bar{\theta})=U^{\dagger}(x, \theta, \bar{\theta}) U(x, \theta, \bar{\theta})=1 .
$$

We emphasize that we have taken into account the fermionic nature (i.e., $\theta C+C \theta=0, \bar{C} \bar{\theta}+\bar{\theta} \bar{C}=0, \theta \bar{C}+\bar{C} \theta=0$, etc.) of the Grassmannian variables $(\theta, \bar{\theta})$ and (anti)ghost fields $(\bar{C}) C$ in the above proof of unitarity. The above SUSP unitary operators can be exponentiated, in a mathematically precise fashion, as follows:

$$
\begin{aligned}
& U(x, \theta, \bar{\theta})=\exp [\theta(-i \bar{C} \cdot T)+\bar{\theta}(-i C \cdot T) \\
& \left.+\theta \bar{\theta}\left(B \cdot T+\frac{(C \times \bar{C})}{2} \cdot T\right)\right], \\
& U^{\dagger}(x, \theta, \bar{\theta})=\exp [\theta(i \bar{C} \cdot T)+\bar{\theta}(i C \cdot T) \\
& \left.+\theta \bar{\theta}\left(-B \cdot T-\frac{(C \times \bar{C})}{2} \cdot T\right)\right] .
\end{aligned}
$$

We mention that $\{C, \bar{C}\}=(C \times \bar{C})$ has been taken into account due to $\left[T^{a}, T^{b}\right]=f^{a b c} T^{c}$ and the fermionic $\left(C^{a} \bar{C}^{b}+\right.$ $\bar{C}^{b} C^{a}=0$ ) nature of the (anti)ghost fields $(\bar{C}) C$. The above exponential form of the unitary operator respects the $\mathrm{SU}(N)$ group structure in the transformation superspace because

$$
\begin{gathered}
\psi(x) \longrightarrow \Psi^{(g)}(x, \theta, \bar{\theta})=\exp [\theta(-i \bar{C})+\bar{\theta}(-i C) \\
\left.+\theta \bar{\theta}\left(B+\frac{(C \times \bar{C})}{2}\right)\right] \psi(x) \equiv U(x, \theta, \bar{\theta}) \psi(x), \\
\bar{\psi}(x) \longrightarrow \bar{\Psi}^{(g)}(x, \theta, \bar{\theta})=\bar{\psi}(x) \exp [\theta(i \bar{C})+\bar{\theta}(i C) \\
\left.+\theta \bar{\theta}\left(-B-\frac{(C \times \bar{C})}{2}\right)\right] \equiv \bar{\psi}(x) U^{\dagger}(x, \theta, \bar{\theta})
\end{gathered}
$$

In other words, we note that the SUSP unitary operators $U(x, \theta, \bar{\theta})$ and $U^{\dagger}(x, \theta, \bar{\theta})$ generate the shift transformations (cf. (10)) along the Grassmannian directions $\theta$ and $\bar{\theta}$ because we note that the ordinary fields $\psi(x)$ and $\bar{\psi}(x)$ are the limiting cases of the superfields $\Psi^{(g)}(x, \theta, \bar{\theta})$ and $\bar{\Psi}^{(g)}(x, \theta, \bar{\theta})$ when the limit is taken to be $\theta=\bar{\theta}=0$ in (14).

The relationship $\psi(x) \rightarrow \Psi^{(g)}(x, \theta, \bar{\theta})=U(x, \theta, \bar{\theta}) \psi(x)$, where the $\operatorname{SU}(N)$ group structure is maintained in the transformation superspace, allows us to define the covariant derivative in terms of the super exterior derivative and super 1 -form connection with the following inherent property for the $4 \mathrm{D}$ non-Abelian 1-form $\mathrm{SU}(N)$ gauge theory:

$$
D \psi(x) \longrightarrow \widetilde{D} \Psi^{(g)}(x, \theta, \bar{\theta})=U(x, \theta, \bar{\theta}) D \psi(x),
$$

where $D \psi(x)=\left(d+i A^{(1)}(x)\right) \psi(x)$ and $\widetilde{D} \Psi^{(g)}(x, \theta, \bar{\theta})=$ $\left(\widetilde{d}+i \widetilde{A}_{(h)}^{(1)}(x, \theta, \bar{\theta})\right) \Psi^{(g)}(x, \theta, \bar{\theta})$. Equation (15) above leads to the following explicit transformation rule for the connection super 1-form in the superspace:

$$
\begin{aligned}
\widetilde{A}_{(h)}^{(1)}(x, \theta, \bar{\theta})= & U(x, \theta, \bar{\theta}) A^{(1)}(x) U^{\dagger}(x, \theta, \bar{\theta}) \\
& +i(\widetilde{d} U(x, \theta, \bar{\theta})) U^{\dagger}(x, \theta, \bar{\theta}) .
\end{aligned}
$$

The substitution of the expressions for $U(x, \theta, \bar{\theta})$ and $U^{\dagger}(x, \theta, \bar{\theta})$, from (11), leads to the following explicit results from the second term of the r.h.s. of (16):

$$
\begin{aligned}
d x^{\mu} & {\left[\theta\left(\partial_{\mu} \bar{C}\right)+\bar{\theta}\left(\partial_{\mu} C\right)+i \theta \bar{\theta}\left(\partial_{\mu} B+\partial_{\mu} C \times \bar{C}\right)\right] } \\
+ & d \theta\left[\bar{C}+\theta\left(-\frac{i}{2}(\bar{C} \times \bar{C})\right)+\bar{\theta}(i B)\right. \\
+ & \theta \bar{\theta}([B, \bar{C}])]+d \bar{\theta}[C+\theta(-i B-i C \times \bar{C}) \\
+ & \left.\bar{\theta}\left(-\frac{i}{2}(C \times C)\right)+\theta \bar{\theta}([B+C \times \bar{C}, C])\right] .
\end{aligned}
$$

Comparing with the coefficients of $(d \theta)$ and $(d \bar{\theta})$ that are present on the 1.h.s. of the super 1-form connection $\left[\widetilde{A}_{(h)}^{(1)}=\right.$ $\left.d x^{\mu} B_{\mu}^{(h)}(x, \theta, \bar{\theta})+d \theta \bar{F}^{(h)}(x, \theta, \bar{\theta})+d \bar{\theta} F^{(h)}(x, \theta, \bar{\theta})\right]$, we observe that we have already derived the correct expressions for the superfields $F^{(h)}(x, \theta, \bar{\theta})$ and $\bar{F}^{(h)}(x, \theta, \bar{\theta})$ where, as is evident, $F^{(h)}(x, \theta, \bar{\theta})$ and $\bar{F}^{(h)}(x, \theta, \bar{\theta})$ are the expansions that have been obtained after the application of the HC. A close look at the coefficients of $d \bar{\theta}$ in (17) shows that if we use the CF-condition $B+\bar{B}+(C \times \bar{C})=0$, we have an alternative form of the last entry in (17); namely,

$$
d \bar{\theta}\left[C+\theta(i \bar{B})+\bar{\theta}\left(-\frac{i}{2}(C \times C)\right)+\theta \bar{\theta}(-\bar{B} \times C)\right] .
$$

This expression (when compared with $d \bar{\theta} F^{(h)}(x, \theta, \bar{\theta})$ from the 1.h.s.) yields the transformations $s_{b} C(x), s_{a b} C(x)$, and $s_{b} s_{a b} C(x)$ as the coefficients of $\theta, \bar{\theta}$, and $\theta \bar{\theta}$, respectively. To fully derive the results of $\mathrm{HC}$, we have to explicitly compute 
the coefficients of $\left(d x^{\mu}\right)$ from the r.h.s. of (16). The coefficient of $d x^{\mu}$, from the sum of the first term and second term on the r.h.s. of transformations (16), yields the following:

$$
\begin{aligned}
d x^{\mu} & {\left[A_{\mu}(x)+\theta\left(D_{\mu} \bar{C}\right)+\bar{\theta}\left(D_{\mu} C\right)\right.} \\
+ & \left.i \theta \bar{\theta}\left(D_{\mu} B+D_{\mu} C \times \bar{C}\right)\right],
\end{aligned}
$$

where the above equation is nothing but the sum of the following:

$$
\begin{aligned}
& d x^{\mu}\left(A_{\mu}+i \theta\left[A_{\mu}, \bar{C}\right]+i \bar{\theta}\left[A_{\mu}, C\right]\right. \\
& \left.\quad+\theta \bar{\theta}\left(-\left[A_{\mu}, B\right]-\left\{\left[A_{\mu}, C\right] \bar{C}\right\}\right)\right), \\
& d x^{\mu}\left[\theta\left(\partial_{\mu} \bar{C}\right)+\bar{\theta}\left(\partial_{\mu} C\right)+i \theta \bar{\theta}\left(\partial_{\mu} B+\partial_{\mu} C \times \bar{C}\right)\right] .
\end{aligned}
$$

We note that contributions (20) come out explicitly from the first and second terms on the r.h.s. of (16). When the above expression is compared with the l.h.s. of the definition $\widetilde{A}_{(h)}^{(1)}=$ $d x^{\mu} B_{\mu}^{(h)}(x, \theta, \bar{\theta})+d \theta \bar{F}^{(h)}(x, \theta, \bar{\theta})+d \bar{\theta} F^{(h)}(x, \theta, \bar{\theta})$, we obtain the expansion for $B_{\mu}^{(h)}(x, \theta, \bar{\theta})$ that has been derived due to HC in (6).

The transformations on $\widetilde{F}^{(2)}=\left[\left(d Z^{M} \wedge d Z^{N}\right) / 2 !\right] \widetilde{F}_{M N}(x$, $\theta, \bar{\theta})$ in the superspace can also be computed by establishing a connection between $\widetilde{F}^{(2)}$ and $F^{(2)}=\left[\left(d x^{\mu} \wedge d x^{\nu}\right) / 2 !\right] F_{\mu \nu}(x)$. In this endeavor, the property of the successive operations of a couple of covariant derivatives and their connection with the curvature 2-form plays an important role. For instance, in the ordinary 4D Minkowskian flat spacetime, we know that

$$
D D \psi(x)=i F^{(2)}(x) \psi(x), \quad D=d+i A^{(1)} .
$$

This relation can be generalized onto $(4,2)$-dimensional supermanifold as

$$
\begin{aligned}
D D \psi & \longrightarrow \widetilde{D} \widetilde{D} \Psi^{(g)}(x, \theta, \bar{\theta}) \\
& =i \widetilde{F}^{(2)}(x, \theta, \bar{\theta}) \Psi^{(g)}(x, \theta, \bar{\theta}),
\end{aligned}
$$

where $\widetilde{D}=\tilde{d}+i A_{(h)}^{(1)}(x, \theta, \bar{\theta})$ and $\Psi^{(g)}(x, \theta, \bar{\theta})=U(x$, $\theta, \bar{\theta}) \psi(x)$. Actually, the relationships of the kind in (21) and (22) are covariant relations [due to $\Psi^{(g)}(x, \theta, \bar{\theta})=$ $U(x, \theta, \bar{\theta}) \psi(x)$ and $\left.\widetilde{D} \Psi^{(g)}(x, \theta, \bar{\theta})=U(x, \theta, \bar{\theta}) D \psi(x)\right]$. Thus, we have the following explicit relationship:

$$
\begin{aligned}
\widetilde{D} \widetilde{D} \Psi^{(g)}(x, \theta, \bar{\theta}) & =i \widetilde{F}^{(2)}(x) U(x, \theta, \bar{\theta}) \psi(x) \\
& \equiv i U(x, \theta, \bar{\theta}) F^{(2)}(x) \psi(x) .
\end{aligned}
$$

The above equation emerges out from (22) [with $\widetilde{D}=$ $U(x, \theta, \bar{\theta}) D U^{\dagger}(x, \theta, \bar{\theta})$ and $\left.\Psi^{(g)}(x, \theta, \bar{\theta})=U(x, \theta, \bar{\theta}) \psi(x)\right]$. From relation (23), it is clear that we have the following explicit relationship:

$$
\widetilde{F}^{(2)}(x, \theta, \bar{\theta})=U(x, \theta, \bar{\theta}) F^{(2)}(x) U^{\dagger}(x, \theta, \bar{\theta}),
$$

which has been mentioned earlier, too, as the transformation property of the curvature $F^{(2)}(x)$ in the superspace.

There is an altogether different theoretical method to derive the transformation property of $F^{(2)}(x)$ in the superspace by exploiting the celebrated Maurer-Cartan type of equation. It will be noted that the exact Maurer-Cartan equation is somewhat different from the relationship that defines the curvature 2-form. However, it looks similar in appearance. From (16), it is evident that we have the following:

$$
\begin{aligned}
\widetilde{d} \widetilde{A}_{(h)}^{(1)}(x, \theta, \bar{\theta})= & (\widetilde{d} U) A^{(1)}(x) U^{\dagger}+U d A^{(1)}(x) U^{\dagger} \\
& -U A^{(1)}(x) \tilde{d} U^{\dagger}-i(\tilde{d} U)\left(\tilde{d} U^{\dagger}\right),
\end{aligned}
$$

where we have used the properties $\tilde{d}\left(U U^{\dagger}\right)=0 \Rightarrow(\tilde{d} U) U^{\dagger}=$ $-U\left(\tilde{d} U^{\dagger}\right), \tilde{d}^{2}=0$, and $\tilde{d} A^{(1)}(x)=d A^{(1)}(x)$. We further observe, from (16), that

$$
\begin{aligned}
i \widetilde{A}_{(h)}^{(1)}(x, \theta, \bar{\theta}) \wedge \widetilde{A}_{(h)}^{(1)}(x, \theta, \bar{\theta}) \\
=U\left(i A^{(1)}(x) \wedge A^{(1)}(x)\right) U^{\dagger}+U A^{(1)}(x)\left(\widetilde{d} U^{\dagger}\right) \\
\quad-(\widetilde{d} U) A^{(1)}(x) U^{\dagger}+i(\widetilde{d} U)\left(\widetilde{d} U^{\dagger}\right),
\end{aligned}
$$

where, once again, we have used the inputs from the differential geometry (i.e., $\left.\tilde{d}^{2}=0\right)$ and $(\tilde{d} U) U^{\dagger}+U\left(\tilde{d} U^{\dagger}\right)=0$. The sum of (25) and (26) yields

$$
\begin{aligned}
& \left(\widetilde{d} \widetilde{A}_{(h)}^{(1)}+i \widetilde{A}_{(h)}^{(1)} \wedge \widetilde{A}_{(h)}^{(1)}\right)=U(x, \theta, \bar{\theta}) \\
& \quad \cdot\left[d A^{(1)}(x)+i A^{(1)}(x) \wedge A^{(1)}(x)\right] U^{\dagger}(x, \theta, \bar{\theta}) .
\end{aligned}
$$

The above relation is basically the relationship $\widetilde{F}^{(2)}(x, \theta, \bar{\theta})=$ $U(x, \theta, \bar{\theta}) F^{(2)}(x) U^{\dagger}(x, \theta, \bar{\theta})$ that has been derived in (24) by exploiting the property of covariant derivatives. Ultimately, we conclude that there are, at least, two different and distinct theoretical methods to compute the relationships $\left(\widetilde{F}^{(2)}(x, \theta, \bar{\theta})=U(x, \theta, \bar{\theta}) F^{(2)}(x) U^{\dagger}(x, \theta, \bar{\theta})\right)$ between $\widetilde{F}^{(2)}(x, \theta, \bar{\theta})$ and $F^{(2)}(x)$ which are the super and ordinary curvature 2 -forms in our $(4,2)$-dimensional SUSY and $(3+$ 1)-dimensional ordinary theories, respectively.

We would like to briefly comment on the relationships between the GIR (invoked in (7)) and the SUSP operators (that have been derived in (11) and (13)). Towards this goal in mind, we focus on the basic definitions of the (super)covariant derivatives that have been quoted in (15). In fact, in its explicit form, this equation can be written as follows:

$$
\begin{aligned}
(\widetilde{d} & \left.+i \widetilde{A}_{(h)}^{(1)}\right) \Psi(x, \theta, \bar{\theta}) \\
& =U(x, \theta, \bar{\theta})\left(d+i A^{(1)}(x)\right) \psi(x) .
\end{aligned}
$$


Using the fundamental definitions of $\widetilde{d}$ and $\widetilde{A}_{(h)}^{(1)}$ from (3) and (16), we obtain the following equalities when we compare the l.h.s. with the r.h.s. of the above equation:

$$
\begin{aligned}
& d x^{\mu}\left[\partial_{\mu} \Psi+i U A_{\mu}(x) U^{\dagger} \Psi-\left(\partial_{\mu} U\right) U^{\dagger} \Psi\right] \\
& =d x^{\mu}\left(U \partial_{\mu} \psi(x)+i U A_{\mu} \psi(x)\right), \\
& d \theta\left[\partial_{\theta} \Psi(x, \theta, \bar{\theta})-\left(\partial_{\theta} U\right) U^{\dagger} \Psi(x, \theta, \bar{\theta})\right]=0, \\
& d \bar{\theta}\left[\partial_{\bar{\theta}} \Psi(x, \theta, \bar{\theta})-\left(\partial_{\bar{\theta}} U\right) U^{\dagger} \Psi(x, \theta, \bar{\theta})\right]=0,
\end{aligned}
$$

where we have used the abbreviations $U \equiv U(x, \theta, \bar{\theta})$ and $U^{\dagger} \equiv U^{\dagger}(x, \theta, \bar{\theta})$. It is quite elementary to observe that the solution $\Psi^{(g)}(x, \theta, \bar{\theta})=U(x, \theta, \bar{\theta}) \psi(x)$ does satisfy the top two equations in (29). It is interesting to note that this solution also satisfies the last equation that is present in (29). If we substitute the exact form of $U(x, \theta, \bar{\theta})$ from (11) into this relationship, we obtain the result in (10) which are derived due to the application of GIR. We further point out that the solution $\Psi^{(g)}(x, \theta, \bar{\theta})=U(x, \theta, \bar{\theta}) \psi(x)$ implies that we also have $\bar{\Psi}^{(g)}(x, \theta, \bar{\theta})=\bar{\psi}(x) U^{\dagger}(x, \theta, \bar{\theta})$. If we substitute the exact mathematical expression for $U^{\dagger}(x, \theta, \bar{\theta})$ from (11) into this relationship, we obtain the (anti-)BRST symmetry transformations for $\bar{\psi}(x)$ field as quoted in (10). Ultimately, we lay emphasis on our key observation that the exact mathematical derivation of the SUSP operators $U$ and $U^{\dagger}$ provides, in some sense, the alternative to $\mathrm{HC}$ as well as GIR (where the SU $(N)$ group structure of the non-Abelian theory is very explicitly maintained in the superspace).

Before we end this section, we would like to lay emphasis on our observation that we have derived the SUSP operators $U(x, \theta, \bar{\theta})$ and $U^{\dagger}(x, \theta, \bar{\theta})$ without invoking any Hermitian conjugation condition from outside on the parameters $(\theta, \bar{\theta})$ and fields $(C, \bar{C}, B)$ of our SUSY theory. However, it can be checked explicitly that if we apply the following Hermitian conjugation operations from outside, namely,

$$
\begin{aligned}
\theta^{\dagger} & =\mp \theta, \\
\bar{\theta}^{\dagger} & =\mp \bar{\theta}, \\
C^{\dagger} & = \pm C, \\
\bar{C}^{\dagger} & = \pm \bar{C}, \\
B^{\dagger} & =B, \\
i^{\dagger} & =-i, \\
(\theta \bar{\theta})^{\dagger} & =\bar{\theta}^{\dagger} \theta^{\dagger}=-\theta \bar{\theta}, \\
(C \bar{C})^{\dagger} & =\bar{C}^{\dagger} C^{\dagger}=\bar{C} C,
\end{aligned}
$$

the SUSP unitary operators interchange (i.e., $U(x, \theta, \bar{\theta}) \leftrightarrow$ $\left.U^{\dagger}(x, \theta, \bar{\theta})\right)$. In other words, by using the above operations, we can obtain $U^{\dagger}(x, \theta, \bar{\theta})$ from $U(x, \theta, \bar{\theta})$ and vice versa. Thus, our theory does support a set of Hermitian conjugate symmetry transformations, too. It should be noted that the Hermitian conjugation conditions, quoted in (30), are not unique. Our theory might support another set of Hermitian conjugation conditions, as well.

\section{Conclusions}

In our present investigation, we have explicitly computed mathematically precise form of the SUSP unitary operator $U(x, \theta, \bar{\theta})$ that appears in the transformation superspace of the Dirac field $\psi(x) \rightarrow \Psi^{(g)}(x, \theta, \bar{\theta})=U(x, \theta, \bar{\theta}) \psi(x)$. We have also computed the Hermitian conjugate SUSP operator (i.e., $\left.U^{\dagger}(x, \theta, \bar{\theta})\right)$ that appears in the transformation of the Dirac field $\bar{\psi}(x) \rightarrow \bar{\Psi}^{(g)}(x, \theta, \bar{\theta})=\bar{\psi}(x) U^{\dagger}(x, \theta, \bar{\theta})$. We have shown that $U U^{\dagger}=U^{\dagger} U=1$ which proves the unitarity of this SUSP operator. The key point, to be noted, is the observation that $U(x, \theta, \bar{\theta})$ and $U^{\dagger}(x, \theta, \bar{\theta})$ appear very naturally from the transformation properties of $\psi(x)$ and $\bar{\psi}(x)$ to $\Psi^{(g)}(x, \theta, \bar{\theta})$ and $\bar{\Psi}^{(g)}(x, \theta, \bar{\theta})$, respectively. We have not invoked any Hermitian conjugation operation from outside on the parameters $(\theta, \bar{\theta})$ and fields $(C, \bar{C}, B$, etc.) of our theory for the computation of $U^{\dagger}(x, \theta, \bar{\theta})$ from the known expression for $U(x, \theta, \bar{\theta})$. However, to demonstrate that our theory also supports a set of Hermitian conjugation operations, we have listed a couple of such operations in (30) on the parameters as well as appropriate fields of our theory which provide a connection between $U(x, \theta, \bar{\theta})$ and $U^{\dagger}(x, \theta, \bar{\theta})$.

The above SUSP transformations (which maintain the $\mathrm{SU}(N)$ group structure) lead to the definition of the covariant derivative on the $(4,2)$-dimensional supermanifold. This definition, in turn, enables us to obtain the transformation $\left(A^{(1)} \rightarrow \widetilde{A}_{(h)}^{(1)}\right)$ on the 1-form connection (cf. (16)). This relationship immediately leads to the derivation of the results of HC. In other words, we derive expansions (6) in terms of the SUSP operators $U(x, \theta, \bar{\theta})$ and $U^{\dagger}(x, \theta, \bar{\theta})$ due to the appearance of (16) in our present endeavor. Thus, the precise derivation of SUSP unitary operators $U$ and $U^{\dagger}$ provides an alternative to the $\mathrm{HC}$ where the group structure is maintained explicitly in the superspace. In fact, these SUSP operators have also enabled us to obtain the results of GIR where, once again, the idea of covariant derivative has played an important role. We have discussed the derivation of the relationships $\Psi^{(g)}(x, \theta, \bar{\theta})=U(x, \theta, \bar{\theta}) \psi(x), \Psi^{(g)}(x, \theta, \bar{\theta})=$ $\bar{\psi}(x) U^{\dagger}(x, \theta, \bar{\theta})$ in equations (28) and (29) (cf. Section 5) where the mathematical forms of SUSP operators $U(x, \theta, \bar{\theta})$ and $U^{\dagger}(x, \theta, \bar{\theta})$ play crucial roles.

As we know, two successive applications of the covariant derivative $D=\left(d+i A^{(1)}\right)$ on the Dirac field $\psi(x)$ (i.e., $D D \psi$ ) lead to the definition of the curvature 2-form $F^{(2)}$ (i.e., $D D \psi=i F^{(2)} \psi$ ). This relation is a covariant relation and one can tap the potential of this relationship to obtain its SUSY version (i.e., $D D \psi \rightarrow \widetilde{D} \widetilde{D} \Psi^{(g)}=i \widetilde{F}^{(2)} \Psi^{(g)}$ ). Now, using the well-known relationship of the covariant 
derivative $\widetilde{D} \Psi^{(g)}=U D \psi$, we obtain the relationship $\widetilde{F}^{(2)} U(x, \theta, \bar{\theta})=U(x, \theta, \bar{\theta}) F^{(2)}(x) \Rightarrow \widetilde{F}^{(2)}(x, \theta, \bar{\theta})=U(x$, $\theta, \bar{\theta}) F^{(2)}(x) U^{\dagger}(x, \theta, \bar{\theta})$ which leads to the derivation of the (anti-)BRST transformations for the curvature tensor $F_{\mu \nu}$ (i.e., $s_{b} F_{\mu \nu}=i\left(F_{\mu \nu} \times C\right)$ and $\left.s_{a b} F_{\mu \nu}=i\left(F_{\mu \nu} \times \bar{C}\right)\right)$ in the context of our $4 \mathrm{D}$ non-Abelian theory. The above precise relationship (i.e., $\left.\widetilde{F}^{(2)}(x, \theta, \bar{\theta})=U(x, \theta, \bar{\theta}) F^{(2)}(x) U^{\dagger}(x, \theta, \bar{\theta})\right)$ reduces to its Abelian counterpart $\widetilde{F}^{(2)}(x, \theta, \bar{\theta})=F^{(2)}(x)$ that has been discussed in our earlier work [11] on the interacting Abelian $U(1)$ gauge theory with Dirac and complex scalar fields where there is an explicit coupling between the gauge field and matter fields.

In our earlier works [18-20], we have derived the nilpotent (anti-)BRST and (anti-)co-BRST symmetry transformations for the Stueckelberg-modified version of the 2D Proca theory [18], the modified version of the $2 \mathrm{D}$ anomalous gauge theory [19], and the 2D self-dual chiral bosonic field theory [20] by exploiting the tools and techniques of the augmented version of geometrical superfield formalism [8-10]. We lay emphasis on the fact that, in these theories [18-20], we have the presence of the matter as well as gauge fields which are coupled to one another in a specific fashion. Thus, it would be very nice idea to find out the SUSP unitary operators for these theories where not only the off-shell nilpotent (anti-)BRST symmetry transformations but also the off-shell nilpotent (anti-)co-BRST symmetries exist, too, for the matter, gauge, and (anti)ghost fields. We are currently intensively involved with these ideas. In this connection, it is gratifying to state that we have partially accomplished this goal in our very recent work [21] where we have discussed only the (anti-)BRST symmetries and corresponding SUSP unitary operators $U(x, \theta, \bar{\theta})$ and $U^{\dagger}(x, \theta, \bar{\theta})$. The discussion about the nilpotent (anti-)co-BRST symmetry transformations and the corresponding dual SUSP unitary operators has been achieved in our very recent work [22]. In this context, it is very gratifying to state that we have also derived the dual SUSP unitary operator and its Hermitian conjugate which lead to the derivation of the proper (anti-)co-BRST symmetry transformations for some of the interesting Abelian models [18-20] of the Hodge theory.

\section{Appendix}

\section{More on the (Anti-)BRST Invariant Lagrangian Densities in the Curci-Ferrari Gauge}

For the readers' convenience, we discuss explicitly a few more key points, connected with the form of our Lagrangian densities $\mathscr{L}_{B}$ and $\mathscr{L}_{\bar{B}}$ in the Curci-Ferrari gauge [cf. (1)] of our present investigation (where we have already taken $\xi=2$ ). It is very interesting to note that (modulo some total spacetime derivatives)

$$
\begin{aligned}
\mathscr{L}_{B}= & -\frac{1}{4} F_{\mu \nu} \cdot F^{\mu \nu}+\bar{\psi}\left(i \gamma^{\mu} D_{\mu}-m\right) \psi \\
& +s_{b} s_{a b}\left(\frac{i}{2} A_{\mu} \cdot A^{\mu}-\bar{C} \cdot C\right),
\end{aligned}
$$

$$
\begin{aligned}
\mathscr{L}_{\bar{B}}= & -\frac{1}{4} F_{\mu \nu} \cdot F^{\mu \nu}+\bar{\psi}\left(i \gamma^{\mu} D_{\mu}-m\right) \psi \\
& -s_{a b} s_{b}\left(\frac{i}{2} A_{\mu} \cdot A^{\mu}-\bar{C} \cdot C\right),
\end{aligned}
$$

where the covariant derivative on the matter field [i.e., $D_{\mu} \psi=$ $\left.\left(\partial_{\mu}+i A_{\mu} \cdot T\right) \psi\right]$ is in the fundamental representation of the $\mathrm{SU}(N)$ Lie algebra. We note that the following are true:

$$
\begin{aligned}
s_{b} s_{a b}\left(\frac{i}{2} A_{\mu} \cdot A^{\mu}\right)= & B \cdot\left(\partial_{\mu} A^{\mu}\right)-i \partial_{\mu} \bar{C} \cdot D^{\mu} C \\
& -\partial_{\mu}\left(A^{\mu} \cdot B\right), \\
-s_{a b} s_{b}\left(\frac{i}{2} A_{\mu} \cdot A^{\mu}\right)= & -\bar{B} \cdot\left(\partial_{\mu} A^{\mu}\right)-i D_{\mu} \bar{C} \cdot \partial^{\mu} C \\
& -\partial_{\mu}\left(A^{\mu} \cdot \bar{B}\right) .
\end{aligned}
$$

Here, the covariant derivative $D_{\mu} C=\partial_{\mu} C+i\left(A_{\mu} \times C\right)$ is in the adjoint representation of the $\mathrm{SU}(N)$ Lie algebra. Thus, we note that the gauge-fixing and Faddeev-Popov ghost terms of the Lagrangian densities $\mathscr{L}_{B}$ and $\mathscr{L}_{\bar{B}}$ are derived from (A.2) modulo a total spacetime derivative term. In the above derivations, we have not used the CF-conditions anywhere.

Now, we concentrate on the computations of the following equivalent expressions:

$$
s_{b} s_{a b}(-\bar{C} \cdot C)=s_{a b} s_{b}(\bar{C} \cdot C),
$$

where we have to use the CF-condition because the absolute anticommutativity $\left(s_{b} s_{a b}+s_{a b} s_{b}\right)=0$ is satisfied only on the hypersurface where $B+\bar{B}+C \times \bar{C}=0$ is true in the $4 \mathrm{D}$ Minkowskian spacetime manifold. In this context, we note that we have the following:

$$
\begin{aligned}
s_{b} s_{a b}(-\bar{C} \cdot C)= & -(B+\bar{B}) \cdot(C \times \bar{C})-B \cdot \bar{B} \\
& +\frac{1}{4}(\bar{C} \times \bar{C}) \cdot(C \times C) \\
\equiv & -(B+\bar{B}) \cdot(C \times \bar{C})-B \cdot \bar{B} \\
& -\frac{1}{2}(C \times \bar{C}) \cdot(C \times \bar{C}) .
\end{aligned}
$$

Now, using the CF-condition $(C \times \bar{C})=-(B+\bar{B})$, we obtain the following:

$$
s_{b} s_{a b}(-\bar{C} \cdot C)=\frac{1}{2}(B \cdot B+\bar{B} \cdot \bar{B}) .
$$

We note that the gauge-fixing and Faddeev-Popov ghost terms of the Lagrangian densities $\mathscr{L}_{B}$ and $\mathscr{L}_{\bar{B}}$ of (1) are nothing other than the appropriate sum of (A.2) and (A.5). It is straightforward to note that $s_{a b} s_{b}(\bar{C} \cdot C)$ produces the same result as in (A.5) (when we use the CF-condition). The (anti-)BRST symmetry transformations $s_{(a) b}$ of the above Lagrangian densities yield the following total spacetime 
derivatives plus terms that are zero on the hypersurface where the CF-condition is valid:

$$
\begin{aligned}
s_{b} \mathscr{L}_{B}= & \partial_{\mu}\left[B \cdot D^{\mu} C\right], \\
s_{a b} \mathscr{L}_{\bar{B}}= & \partial_{\mu}\left[-\bar{B} \cdot D^{\mu} \bar{C}\right], \\
s_{a b} \mathscr{L}_{B}= & \partial_{\mu}\left[-\{\bar{B}+(C \times \bar{C})\} \cdot \partial^{\mu} \bar{C}\right] \\
& +(B+\bar{B}+C \times \bar{C}) \cdot D_{\mu}\left(\partial^{\mu} \bar{C}\right) \\
\equiv & \partial_{\mu}\left[B \cdot \partial^{\mu} \bar{C}\right]+(B+\bar{B}+C \times \bar{C}) \\
& \cdot D_{\mu}\left(\partial^{\mu} \bar{C}\right), \\
s_{b} \mathscr{L}_{\bar{B}}= & \partial_{\mu}\left[\{B+(C \times \bar{C})\} \cdot \partial^{\mu} C\right] \\
& -(B+\bar{B}+C \times \bar{C}) \cdot D_{\mu}\left(\partial^{\mu} C\right) \\
\equiv & \partial_{\mu}\left[-\bar{B} \cdot \partial^{\mu} C\right]-(B+\bar{B}+C \times \bar{C}) \\
& \cdot D_{\mu}\left(\partial^{\mu} C\right) .
\end{aligned}
$$

Thus, as far as the symmetry properties are concerned, the Lagrangian densities $\mathscr{L}_{B}$ and $\mathscr{L}_{\bar{B}}$ are equivalent on the hypersurface (in the 4D Minkowskian spacetime manifold) where the CF-condition $B+\bar{B}+C \times \bar{C}=0$ is satisfied. In fact, the absolute anticommutativity property $\left(s_{b} s_{a b}+s_{a b} s_{b}=0\right)$ of the (anti-)BRST symmetry transformations $s_{(a) b}$ is also true only on this hypersurface. We end this Appendix with the remark that the Lagrangian densities $\mathscr{L}_{B}$ and $\mathscr{L}_{\bar{B}}$ can be expressed as follows:

$$
\begin{aligned}
\mathscr{L}_{B}= & -\frac{1}{4} F^{\mu \nu} \cdot F_{\mu \nu}+\bar{\psi}\left(i \gamma^{\mu} D_{\mu}-m\right) \psi+B \cdot\left(\partial_{\mu} A^{\mu}\right) \\
& +B \cdot B+B \cdot(C \times \bar{C})+\frac{1}{2}(C \times \bar{C}) \\
& \cdot(C \times \bar{C})-i \partial_{\mu} \bar{C} \cdot D^{\mu} C, \\
\mathscr{L}_{\bar{B}}= & -\frac{1}{4} F^{\mu \nu} \cdot F_{\mu \nu}+\bar{\psi}\left(i \gamma^{\mu} D_{\mu}-m\right) \psi-\bar{B} \cdot\left(\partial_{\mu} A^{\mu}\right) \\
& +\bar{B} \cdot \bar{B}+\bar{B} \cdot(C \times \bar{C})-\frac{1}{2}(C \times \bar{C}) \\
& \cdot(C \times \bar{C})-i D_{\mu} \bar{C} \cdot \partial^{\mu} C .
\end{aligned}
$$

The equations of motion (i.e., $\partial \mathscr{L}_{B} / \partial B=0$ and $\partial \mathscr{L}_{\bar{B}} / \partial \bar{B}=0$ ) with respect to $B$ and $\bar{B}$ yield the following:

$$
\begin{aligned}
\partial_{\mu} A^{\mu}+2 B+(C \times \bar{C}) & =0, \\
-\partial_{\mu} A^{\mu}+2 \bar{B}+(C \times \bar{C}) & =0 .
\end{aligned}
$$

The sum of the above relationships leads to the derivation of the CF-condition.

\section{Competing Interests}

The authors declare that they have no competing interests.

\section{Acknowledgments}

One of the authors (R. P. Malik) would like to express his deep sense of gratitude to the AS-ICTP and SISSA, Trieste, Italy, for the warm hospitality extended to him during his participation in the conference on "Aspects of Gauge and String Theories"(1-2 July 2015) which was held at SISSA to celebrate the 70th birth anniversary of L. Bonora. The idea behind this work came during this conference. D. Shukla is thankful to the UGC, Government of India, New Delhi, for financial support through RFSMS-SRF scheme and T. Bhanja is grateful to the $\mathrm{BHU}$-fellowship under which the present investigation has been carried out. Fruitful comments by our esteemed reviewer are gratefully acknowledged, too.

\section{References}

[1] L. Bonora and M. Tonin, "Superfield formulation of extended BRS symmetry," Physics Letters B, vol. 98, no. 1-2, pp. 48-50, 1981.

[2] L. Bonora, P. Pasti, and M. Tonin, "Extended BRS symmetry in non-Abelian gauge theories," Il Nuovo Cimento A, vol. 64, no. 3 , pp. 307-331, 1981.

[3] L. Bonora, P. Pasti, and M. Tonin, "Superspace approach to quantum gauge theories," Annals of Physics, vol. 144, no. 1, pp. $15-33,1982$.

[4] R. Delbourgo and P. D. Jarvis, "Extended BRS invariance and OSp (4/2) supersymmetry supersymmetry," Journal of Physics A: Mathematical and General, vol. 15, no. 2, p. 611, 1982.

[5] R. Delbourgo, P. D. Jarvis, and G. Thompson, "Local OSp(4/2) supersymmetry and extended BRS transformations for gravity," Physics Letters B, vol. 109, no. 1-2, pp. 25-27, 1982.

[6] L. Bonora and R. P. Malik, "BRST, anti-BRST and gerbes," Physics Letters B, vol. 655, no. 1-2, pp. 75-79, 2007.

[7] L. Bonora and R. P. Malik, "BRST, anti-BRST and their geometry," Journal of Physics. A. Mathematical and Theoretical, vol. 43, no. 37, 375403, 17 pages, 2010.

[8] R. Malik and B. Mandal, "Augmented superfield approach to exact nilpotent symmetries for matter fields in non-Abelian theory," The European Physical Journal C, vol. 47, no. 1, pp. 219226, 2006.

[9] R. R. Malik, "Augmented superfield approach to unique nilpotent symmetries for complex scalar fields in QED," The European Physical Journal C, vol. 48, no. 3, pp. 825-834, 2006.

[10] R. P. Malik, "Superfield approach to nilpotent symmetries for QED from a single restriction: an alternative to the horizontality condition," Journal of Physics. A. Mathematical and General, vol. 39, no. 33, pp. 10575-10587, 2006.

[11] D. Shukla, T. Bhanja, and R. P. Malik, "Superspace unitary operator in QED with Dirac and complex scalar fields: superfield approach," Europhysics Letters, vol. 112, no. 1, Article ID 11001, 2015.

[12] N. Nakanishi and I. Ojima, Covariant Operator Formalism of Gauge Theories and Quantum Gravity, World Scientific, Singapore, 1990.

[13] K. Nishijima, "BRS-invariance, asymptotic freedom and color confinement (a review)," Czechoslovak Journal of Physics, vol. 46, no. 1, pp. 1-40, 1996.

[14] D. Dudal, V. E. R. Lemes, M. S. Sarandy, S. P. Sorella, and M. Picariello, "On the $\mathrm{SL}(2, \mathbb{R})$ symmetry in yang-mills theories in 
the landau, curci-ferrari and maximal abelian gauge," Journal of High Energy Physics, vol. 2002, no. 12, article 8, 2002.

[15] D. Dudal, H. Verschelde, V. E. R. Lemes et al., "More on ghost condensation in Yang-Mills theory: BCS versus Overhauser effect and the breakdown of the Nakanishi-Ojima annex SL(2,R) symmetry," Journal of High Energy Physics, vol. 2003, no. 6, article 003, 2003.

[16] G. Curci and R. Ferrari, "Slavnov transformations and supersummetry," Physics Letters B, vol. 63, no. 1, pp. 91-94, 1976.

[17] R. P. Malik and B. P. Mandal, "Augmented superfield approach to exact nilpotent symmetries for matter fields in non-abelian theory," The European Physical Journal C. Particles and Fields, vol. 47, no. 1, pp. 219-226, 2006.

[18] T. Bhanja, D. Shukla, and R. P. Malik, "Novel symmetries in the modified version of two dimensional Proca theory," The European Physical Journal C, vol. 73, no. 8, article 2535, 2013.

[19] S. Gupta, R. Kumar, and R. P. Malik, "On free 4D Abelian 2form and anomalous 2D Abelian 1-form gauge theories," The European Physical Journal C, vol. 65, no. 1-2, pp. 311-329, 2010.

[20] D. Shukla, T. Bhanja, and R. P. Malik, "Self-dual chiral boson: augmented superfield approach," The European Physical Journal C, vol. 74, article 3025, 2014.

[21] T. Bhanja, N. Srinivas, and R. P. Malik, "Universal superspace unitary operator for some interesting abelian models: superfield approach," Advances in High Energy Physics, vol. 2016, Article ID 3673206, 11 pages, 2016.

[22] T. Bhanja, N. Srinivas, and R. P. Malik, "Universal superspace unitary operator and nilpotent (anti-)dual BRST symmetries: superfield formalism," http://arxiv.org/abs/1603.08104. 

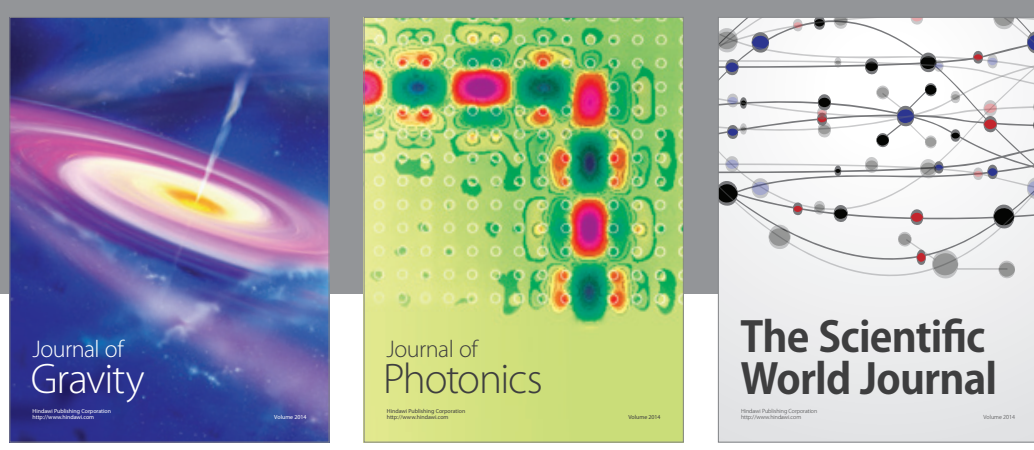

The Scientific World Journal
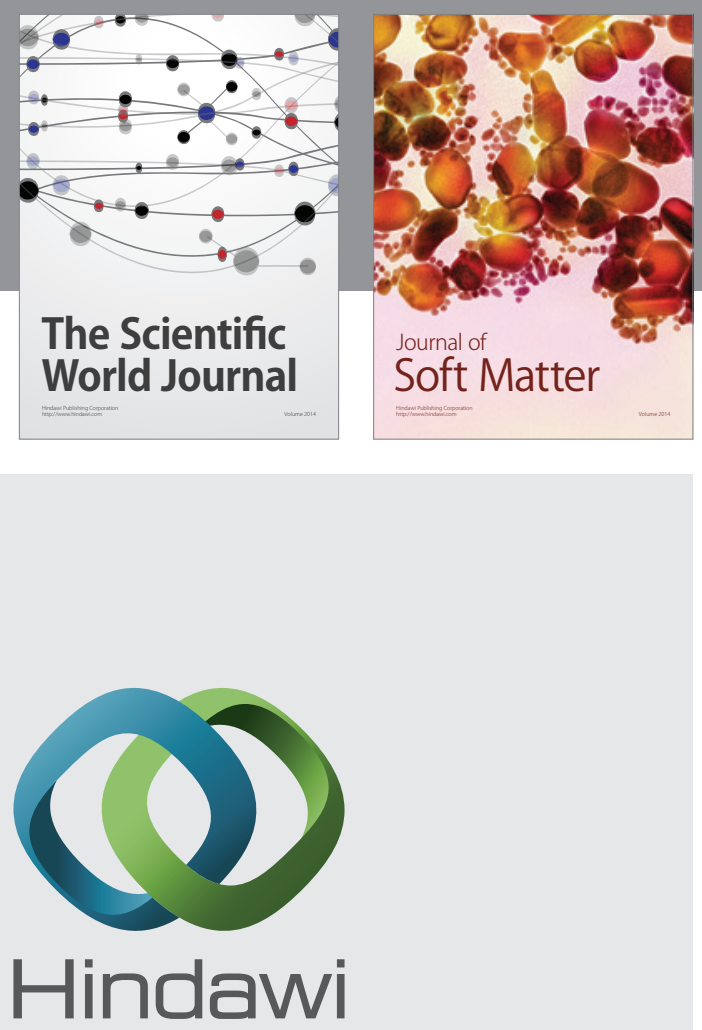

Submit your manuscripts at

http://www.hindawi.com

nternational Journal of

Statistical Mechanics
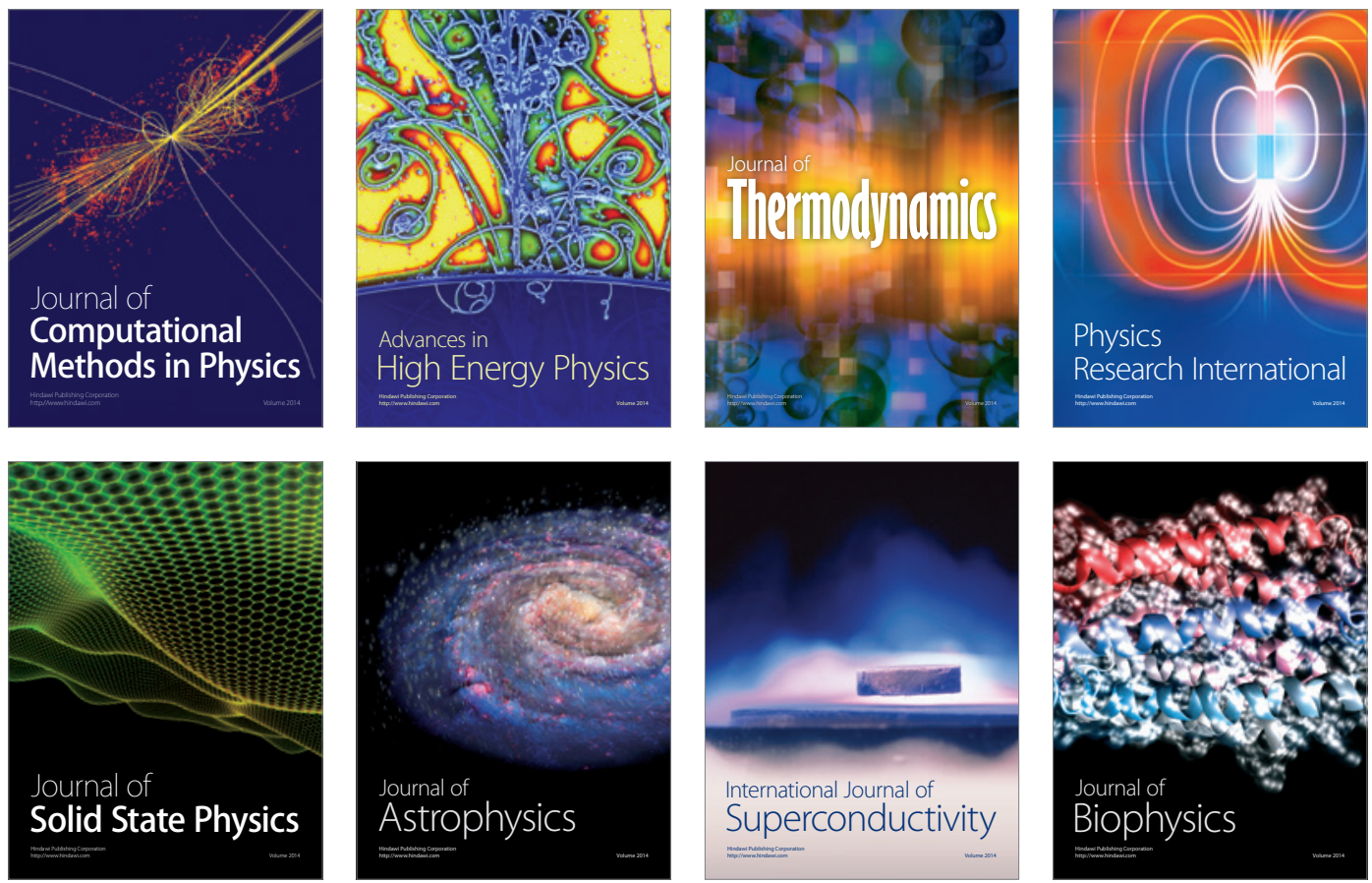
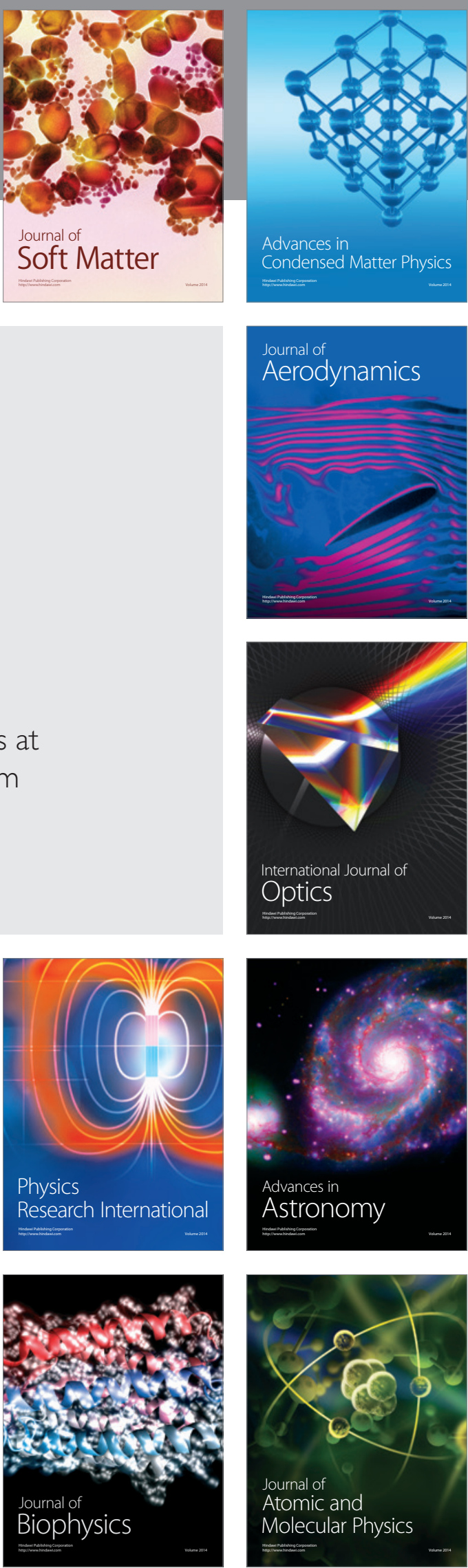